\title{
HIPERPLASIA EPITELIAL FOCAL: UMA REVISÃO DE LITERATURA NARRATIVA
}

\section{HYPERPLASIA EPITHELIAL FOCAL: A NARRATIVE LITERATURE REVIEW}

\author{
Beatriz de Melo Curvina Rosa ${ }^{1}$; Jacqueline Vitória do Nascimento Azevedo²; \\ Nicolle Santos Silva Cutrim ${ }^{3}$; Cyrene Piazera Silva Costa ${ }^{4}$
}

\begin{abstract}
RESUMO: O objetivo deste estudo foi descrever a doença Hiperplasia Epitelial Focal (HEF), caracterizando sua aparência e o seu diagnóstico, a partir de uma revisão de literatura. A HEF afeta mais as meninas. Clinicamente, essa doença apresenta-se como nódulos e/ou pápulas múltiplas de coloração igual da mucosa normal localizados lábios, mucosa jugal e língua. Esta doença está diretamente relacionada com a infecção pelo Papiloma Vírus Humano sendo relacionada no contexto do abuso sexual infantil. E por fim, para que o diagnóstico da HEF possa ser feito, o cirurgião dentista deve estar atento ao exame clínico e aos dados sócio-demográficos do paciente, junto com exames histológicos e o tipo de HPV. Portanto, é importante conhecer tal patologia.
\end{abstract}

PALAVRAS-CHAVE: Hiperplasia epitelial focal. Doença de Heck. Papiloma vírus Humano (HPV).

ABSTRACT: Objective of this study was to describe the Focal Epithelial Hyperplasia (HEF) disease, characterizing its appearance and its diagnosis, based on a review of narrative literature. HEF affects girls more. Clinically this disease presents as nodules and / or multiple papules of equal coloration of the normal mucosa located lips, jugal mucosa and tongue. This disease is directly related to Human Papilloma Virus infection being related in the context of child sexual abuse. Finally, in order for the HEF diagnosis to be made, the dental surgeon must be attentive to the clinical examination and the patient's socio-demographic data, together with histological exams and the type of HPV. Therefore, it is important to know this pathology.

KEYWORDS: Focal epithelial hyperplasia; Heck's disease; Human Papillomavirus (HPV).

\footnotetext{
1 Discente do curso de Odontologia, $2^{\circ}$ período, Liga Acadêmica de Qualidade de Vida - LAQUAVI, Universidade CEUMA. E-mail: beatriz.melo0607@icloud.com

${ }^{2}$ Discente do curso de Odontologia, $2^{\circ}$ período, Liga Acadêmica de Qualidade de Vida - LAQUAVI, Universidade CEUMA. E-mail: duartejacque@outlook.com

${ }^{3}$ Discente do curso de Odontologia, $2^{\circ}$ período, Liga Acadêmica de Qualidade de Vida - LAQUAVI, Universidade CEUMA. E-mail: nicollecutrim@ hotmail.com

4 Docente do curso de Odontologia, coordenadora da Liga Acadêmica de Qualidade de Vida - LAQUAVI, Universidade CEUMA. E-mail: cyrenepiazera@ hotmail.com
} 


\section{INTRODUÇÃO}

Hiperplasia Epitelial Focal (HEF) ou doença de Heck é uma enfermidade rara em todo o mundo (DOS SANTOS et al., 2004; GONZÁLES et al., 2005; LEDESMA-MONTES; GARCÉSORTIZ; HERNÁNDEZ-GUERRERO, 2007). A sua frequência apresenta uma grande variação de uma área geográfica para outra e pode variar, de acordo com Clausen (1975), 0,002 a 35\%, dependendo da população estudada.

O Papiloma vírus Humano (HPV) tem sido apontado como um fator etiológico desta proliferação benigna assintomática da mucosa bucal, principalmente o genótipo 32 (HPV 32) em pessoas de mais idade, e o genótipo 13 (HPV 13), que parece estar igualmente envolvido no desenvolvimento da doença tanto em pacientes jovens quanto nas idades mais avançadas (PADAYACHEE; VAN WYK, 1991; JABLONSKA, 2000). Ainda, têm também sido envolvidos fatores como condições socio-econômicas e genéticos (CARLOS; SEDANO, 1994; SAID et al., 2012).

A HEF é comumente encontrada no sexo feminino, em crianças e adolescentes de baixa condição sócio-econômica, esquimós e índios residentes no continente americano, com ocorrência intrafamiliar (DIAZ et al., 2009). A HEF clinicamente caracteriza-se por nódulos e/ou papulas, únicos ou múltiplos, com tamanho variando entre 0.1 a $1 \mathrm{~cm}$ (LEDESMA; GARCÉS; HERNÁNDEZ, 2007).

Embora o diagnóstico da HEF possa ser feito por meio do exame clínico somado ao levantamento dos dados sócio demográficos do paciente, o diagnóstico definitivo sempre é realizado por meio do exame histológico e detecção do tipo de HPV. Muitas vezes, esta doença possui regressão espontânea. O tratamento é indicado quando as suas lesões atrapalham estética e funcionalmente o paciente. Não existe uma terapia específica para HEF, embora a remoção cirúrgica, excisão a laser ou o uso tópico de agentes antivirais sejam indicadas. (GONZALES et al., 2005).

Desta forma, é importante conhecer tal patologia, uma vez que, para o diagnóstico da HEF possa ser feito, o cirurgião-dentista deve estar atento ao exame clínico e aos dados sóciodemográficos do paciente, junto com exames histológicos e o tipo de HPV. Neste contexto, o objetivo deste estudo foi descrever a doença HEF, caracterizando sua aparência e o seu diagnóstico. 


\section{MATERIAIS E MÉTODOS}

Para a realização desta revisão de literatura narrativa, foi consultada a base de dados PubMed e Google acadêmico utilizando os descritores focal epithelial hyperplasia (hiperplasia epitelial focal), Heck's illness (doença de Heck) e oral manifestations (manifestações orais). Esta seleção foi realizada por um único examinador, inicialmente feito pela leitura dos resumos, seguida pela leitura completa dos artigos.

\section{RESULTADOS E DISCUSSÃO}

Por todo o mundo, a HEF é rara e entretanto, frequentemente é observada nas comunidades pobres, indígenas e de esquimós residentes no continente americano. Nestas populações, comumente as crianças e pessoas do sexo feminino são as mais afetadas (DOS SANTOS et al., 2004; GONZÁLES et al., 2005; LEDESMA-MONTES; GARCÉS-ORTIZ; HERNÁNDEZGUERRERO, 2007). Em um estudo transversal das alterações da mucosa bucal entre 587 indígenas Waimiri-Atroari, habitantes da região amazônica central brasileira, não miscigenados, mantenedores de seus costumes sociais e modo de vida tradicionais, e livres de hábitos como o tabagismo e alcoolismo, a HEF foi a segunda desordem mais comum da mucosa bucal, afetando $13.3 \%$ das crianças e $8.6 \%$ dos adultos (DOS SANTOS et al., 2004). Ainda, segundo Gonzáles et al. (2005) e Ledesma-Montes, Garcés-Ortiz e Hernández-Guerrero (2007), as mulheres possuem cinco vezes mais chance de apresentarem a doença do que os homens.

Apesar da condição sócio-econômica está relacionada com a origem da HEF, é apenas um fator modulador dessa doença (CARLOS; SEDANO, 1994; SAID et al., 2013). Na literatura, o HPV que tem sido apontado como um fator etiológico da HEF, principalmente o genótipo 32 (HPV 32), com tendência a causar a doença em pessoas de mais idade, e o genótipo 13 (HPV 13), que parece estar igualmente envolvido no desenvolvimento da doença tanto em pacientes jovens quanto nas idades mais avançadas (PADAYACHEE; VAN WYK, 1991; JABLONSKA, 2000).

Nos estudos de García-Corona et al. (2004), Gonzáles et al. (2005), Cubero et al. (2006); Lopez-Villanueva et al. (2011), o HPV 13 foi detectado em 100\% das lesões de HEF examinadas e sempre destacam a ocorrência intrafamiliar. Outro fator etiológico importante é a predisposição genética (GARCÍA-CORONA et al., 2004). Conforme García-Corona et al. (2004), o aumento da prevalência da HEF pode estar relacionado com a presença do alelo HLA-DRB1*0404. Segundo Vargas-Alarcon et al. (2000), o alelo HLA-DR é comum em certos grupos étnicos descendentes de 
indígenas americanos, tais como Mazatecans, Nahuas e populações mestiças mexicanas, assim se registra um aumento da evidência de que exista um componente genético na determinação desta doença.

O diagnóstico clínico definitivo desta doença sempre deve ser feito com ajuda de uma anamnese apurada somada ao exame clínico, histopatológico e a tipagem do HPV (GONZÁLES et al., 2005). Nas crianças, é muito importante fazer o diagnóstico diferencial da HEF do Condiloma Acuminado (CA), que é uma doença sexualmente transmissível e indica que o paciente infantil pode estar sofrendo de abuso sexual (COHEN; HEBER; ADLER-STORTHZ; 1993; GUILL et al, 2002). Embora o CA seja clinicamente muito parecido com a HEF, esta patologia geralmente esta associada ao HPV 6 e 11 (EVERSOLE et al., 1987; HENLEY; SUMMERLIN; TOMICH, 2004).

Clinicamente, esta doença geralmente caracteriza-se pela ocorrência de nódulos e/ou pápulas, únicos ou múltiplos, com tamanho variando entre 0.1 a $1 \mathrm{~cm}$, com coloração igual da mucosa normal, podendo ser também branca, não doloroso, propenso a se agrupar e formar verrugas nos lábios, na mucosa jugal e língua (GONZÁLES et al., 2005; GONZÁLES-LOSA et al., 2011; LOPEZ-VILLANUEVA et al., 2011). A sua apresentação clínica pode ser influenciada pela idade do paciente. Lesões múltiplas, exofílicas e nodulares da HEF afetam mais pacientes jovens, enquanto os mais velhos são afetados por uma única lesão papular (GONZÁLES et al., 2005). Além disso, esta doença raramente localiza-se em gengiva e palato duro (LEDESMA-MONTES, GARCÉS-ORTIZ E HERNÁNDEZ-GUERRERO, 2007).

A HEF apresenta características histopatológicas distintas. São elas: proliferação epitelial com acantose proeminente, alterações epiteliais, como presença de coilócitos, células mitosóides ocasionais e focos hiperplásicos com projeções confluentes para o tecido conjuntivo, sendo algumas dessas em forma de "taco de golfe" (VERA-IGLESIAS et al., ).

Por fim, o tratamento desta doença nem sempre é indicado, uma vez que as suas lesões são assintomáticas, regridem espontâneamente e não sofrem malignização. Entretanto lesões que atrapalham o paciente estética e funcionalmente devem ser eliminadas. A literatura destaca, como terapias das lesões de HEF, interferon tópico ou sistêmico, imiquimode creme a $5 \%$ e retinoide sistêmico associado ou não a cirurgia ou eletrocoagulação (VILMER et al., 1994; KÖSE ET A., 200; MASCHKE; BRAUNS; GOOS, 2004). 


\section{CONCLUSÃO}

A HEF clinicamente caracteriza-se por nódulos e/ou papulas, únicos ou múltiplos, com tamanho variando entre 0.1 a $1 \mathrm{~cm}$ assintomáticas e de regressão espontânea, geralmente encontrada em meninas de uma mesma família de baixa condição sócio econômica, esquimós e índios americanos. Apesar da HEF ser uma doença rara, apresenta grande relevância clínica dentro do contexto do abuso sexual infantil. Por isso, o cirurgião-dentista deve conhecê-la, buscando diagnóstico definitivo por meio do exame histológico e detecção do tipo de HPV.

\section{REFERÊNCIAS}

CARLOS, R.; SEDANO, H.O. Multifocal papilloma virus epithelial hyperplasia. Oral. Surg. Med. Pathol., v.77, p. 631-635, 1994.

CLAUSEN, F.P. Geographical aspects of oral focal epithelial hyperplasia. Phat.Microbio., v.39, p.204-213, 1975

COHEN, P.R., HEBERT, A.A.; ADLER-STORTHZ, K. Focal epithelial hyperplasia: Heck disease. Pediatr. Dermatol.,v.10, p. 245-251, 1993.

CUBERO V. ; PEREZ J. ; LOPEZ C. J. et al. Molecular and serological evidence of the epidemiological association of HPV 13 with focal epithelial hyperplasia: A case-control study. J. Clin. Virol., v. 37, n. 1, p. 21-6, 2006.

DOS SANTOS P. J.; BESSA C. F.; DE AGUIAR M. C. et al. Cross-sectional study of oral mucosal conditions among a central Amazonian Indian community, Brazil. J. Oral. Pathol. Med., v. 33, p. 7-12, 2004

EVERSOLE, L.R.; LAIPIS, P.J.; MERRELL, P.; et al. Demonstration of human papillomavirus DNA in oral condyloma acuminatum. J. Oral Pathol., v.16, p. 266-272, 1987.

DÍAZ C.X.O.; GONZÁLEZ S.; DONATTI, L.; et al. Hiperplasia epitelial focal - Enfermedad de Heck: Nuestra casuística. Arch. Argent. Dermatol., v. 59, n. 5, p. 193-199, 2009. 
GARCÍA-CORONA, C.; VEGA-MEMIJE, E.; MOSQUEDA-TAYLOR, A.; et al. Association of HLA-DR4 (DRB1*0404) with human papillomavirus infection in patients with focal epithelial hyperplasia. Arch. Dermatol., v.140, n.10, p. 1227-12231, 2004.

GONZÁLEZ-LOSA, M.R.; SUAREZ-ALLÉN, R.E.; CANIL-CANINE, J.; et al. Multifocal epithelial hyperplasia in a community in the Mayan area of Mexico. Int. J. Dermatol., v. 50, n. 3, p. 304-9, 2011.

GONZALES, L.V.; GAVIRIA, A.M.; SAN CLEMENTE, G.; et al. Clinical, histopathological and virological findings in patients with focal epithelial hyperplasia from Colombia. Int. J. Dermatol., v. 44, n. 4, p. 274-9, 2005.

GUILL, C.K.; HWANG, L.Y.; IYENGAR, V.; et al. Asymptomatic labial papules in a teenager. Arch. Dermatol., v. 138, p. 1509-1514.

FREITAS, R.A.; COSTA, A.L.L.; MEDEIROS, A.M. C.; et al. Hiperplasia epitelial focal (doença de Heck). J. Bras. Patol. Med. Lab, v. 43, n. 6 Rio de Janeiro, 2007.

HENLEY, J.D.; SUMMERLIN, D.J.; TOMICH, C,E. Condyloma acuminatum and condylomalike lesions of the oral cavity: a study of cases with an intraductal component. Histopathology, v.44, p. 216-221, 2004.

JABLONSKA, S. Demonstration of hpv 24 in long-standing Heck's disease with malignant transformation. Eur. J. Dermatol., v. 10, n. 3, p. 235-6, 2000

LEDESMA-MONTES, C.; GARCÉS-ORTIZ, M.; HERNÁNDEZ-GUERRERO, J.C.

Clinicopathological and immunocytochemical study of multifocal epithelial hyperplasia. J. Oral Maxillofac. Surg., v. 65, n. 11, p. 2211-7, 2007.

LOPEZ-VILLANUEVA, M.E.; CONDE-FERRÁEZ, L.; AYORA-TALAVERA, G.; et al. Human papillomavirus 13 in a Mexican Mayan community with multifocal epithelial hyperplasia: could saliva be involved in household transmission. Eur. J. Dermatol., v. 21, n. 3, p. 396-400, 2011. 
MASCHKE, J.; BRAUNS, T.C.; GOOS, M. Imiquimod for the topical treatment of focal epithelial hyperplasia (Heck disease) in a child. J. Dtsch. Dermatol. Ges., v. 2, n. 10, p. 848-50, 2004.

PADAYACHEE, A.; VAN WYK, C.W. Human papillomavirus (HPV) DNA in focal epithelial hyperplasia by in situ hybridization. J. Oral Pathol. Med., v. 20, p. 210-214, 1991.

SAID, A.K.; LEAO, J.C.; FEDELE, S.; et al. Focal eplthellal hypeplsia - an update. J Oral Pathol Med, v. 2, n. 7, p. 293-296, 2012.

VARGAS-ALARCON, G.; GAMBOA, R.; ZUNIGA, J.; et al. HLA-DR4allele frequencies on Indian and Mestizo population from Mexico. Hum. Immunol., v.61, p.341-344, 2000.

VERA-IGLESIAS, E.; GARCÍA-ARPA, M.; SÁNCHEZ-CAMINERO, P.; et al.. Hiperplasia epitelial focal. Actas Dermosifiliogr., v. 98, n. 9, p. 621-3, 2007. 